\title{
Theory of Ostwald ripening for open systems ${ }^{a)}$
}

\author{
C. W. J. Beenakker) and John Ross \\ Department of Chemistry, Stanford University, Stanford, California 94305
}

(Received 21 June 1985; accepted 26 July 1985)

\begin{abstract}
Diffusion-controlled coarsening (Ostwald ripening) of a precipitate is analyzed for the case of an open system, immersed in a reservoir of constant solute concentration. Equivalence of the evolution of such open systems and closed systems studied previously is established in the limit of infinite systems. The cause of this equivalence is screening of the bulk of the system from the reservoir by diffusive interactions between the precipitated particles. The applicability to large, but finite systems is discussed.
\end{abstract}

\section{INTRODUCTION}

Ostwald ripening, or coarsening, of a precipitate is the final stage of phase separation in a solution, during which the larger particles of the precipitate grow at the expense of the smaller particles, which disappear. As recognized by Ostwald,${ }^{1}$ the driving force for the process which now bears his name is the increased solubility of the smaller particles due to surface tension between the precipitate and the solute. If we assume that the solute is in local equilibrium with the precipitate, then this solubility difference induces a solute concentration gradient and leads to a diffusive flux from the smaller to the larger particles. One speaks of diffusion-controlled growth (as opposed to growth controlled by slow deposition of solute atoms at the particle surfaces).

The theory of Ostwald ripening began with the works of Lifshitz and Slyosov ${ }^{2}$ and of Wagner. ${ }^{3}$ Since then a detailed theoretical understanding of this phenomenon has been obtained (see the recent review article by Voorhees ${ }^{4}$ ). The present paper deals with one aspect of the problem which, however, has received only little attention. This is the issue of the process occurring in closed vs open systems.

The assumption of a closed system plays an essential role in the Lifshitz-Slyosov-Wagner theory ${ }^{2,3}$ (and subsequent theories, see Ref. 4), since it allows a determination of the (average) solute concentration from properties of the precipitate, by invoking the law of conservation of mass in the closed system. Obviously, such a procedure is not possible in the case of an open system in contact with a reservoir, since the possibility of transport of solute between the system and the reservoir has to be accounted for (cf. Ref. 5). What, then, is the status of the theory of Ostwald ripening for open systems? We investigate this issue here for the case of an open system immersed in a reservoir of constant solute concentration.

In Sec. II we formulate the equations which determine the coarsening of the precipitate on a microscopic level of description. ${ }^{4,6-8}$ The solutions of these equations are determined by a "transport matrix," which is symmetric and positive definite and which in the limit of an infinite system satisfies a certain sum rule. These properties of the transport matrix, proven in Sec. III, reflect the tendency toward minimalization of the surface to volume ratio of the precipitate, as well as a drastic decrease of the effective range of diffusive

\footnotetext{
a) This work has been supported in part by the National Science Foundation and the Air Force Office of Scientific Research.

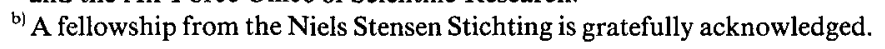

couplings (screening ${ }^{9}$ ). In Sec. IV the equivalence of Ostwald ripening in open and closed infinite systems is established, on the basis of the results of the previous section. In large, but finite systems this equivalence is estimated to hold for periods much longer than the characteristic coarsening time scale, provided the size of the system is initially much greater than the screening length of diffusive interactions (typically of the order of the interparticle separation).

We conclude in Sec. $V$ with a direct microscopic derivation of Wagner's ${ }^{3}$ mean-field evolution equation for the particle-size distribution function in dilute open systems.

\section{FORMULATION OF THE THEORY}

Consider a solution in which precipitated particles grow or dissolve by diffusion of solute through the solution. The basic assumptions of the theory of Ostwald ripening in twophase systems are summarized by Voorhees. ${ }^{4}$

The precipitate is assumed to consist of immobile spherical particles with position vectors $\mathbf{R}_{i}$ and radii $a_{i}(t)$ $(i=1,2, \ldots, N)$ which evolve in time according to

$$
4 \pi a_{i}^{2} \frac{d a_{i}}{d t}=-v Q_{i}, \quad i=1,2, \ldots, N .
$$

Here $v$ is the molar volume of the precipitate and $Q_{i}$ is the integral over the surface of particle $i$ of the molar solute flux. In terms of the molar concentration field $c(\mathbf{r}, t)$ of the solute we have

$$
Q_{i}=-D \oint_{S_{i}} d S \hat{n}_{i} \cdot \nabla c
$$

where $D$ is the diffusion coefficient of the solute, $S_{i}$ the surface of particle $i$, and $\hat{n}_{i}$ a unit vector perpendicular to that surface and pointing outward.

The solute concentration field is determined from the quasistatic diffusion equation

$$
D \nabla^{2} c=0,
$$

supplemented by local equilibrium boundary on the surfaces of the particles

$$
c=c_{\mathrm{eq}}\left(a_{i}\right)=c_{\infty}\left(1+d / a_{i}\right) \quad \text { on } S_{i} .
$$

The solute concentration in equilibrium with a plane surface is denoted by $c_{\infty}$, and $d$ denotes the capillary length. (In a typical solution $d \sim 10^{-7} \mathrm{~cm} .{ }^{3}$ ) We consider the case of an open system immersed in a solute reservoir of constant concentration $c_{0}$, so that we have an additional boundary condition "at infinity," 


$$
c \rightarrow c_{0}=\text { constant, far from particles. }
$$

Use of the quasistatic approximation to the diffusion equation [Eq. (3)] is justified if the characteristic relaxation time $\tau_{D}$ of the diffusive field is much shorter than the coarsening time scale $\tau_{C}=a^{3} / D v c_{\infty} d$ [Ref. 3, Eq. (VI.11)], in which the particle-size distribution function changes appreciably ( $a$ denotes the average particle radius). We estimate $\tau_{D}=a^{2} / D \phi$; with $\phi$ the volume fraction of the precipitate; this is the time it takes a perturbation of the concentration to diffuse over a distance of the order of the effective range $\lambda \approx a / \sqrt{\phi}$ of the diffusive interactions. [For this estimate of the screening length $\lambda$, see Eq. (29) in Sec. III.] The requirement $\tau_{D} \ll \tau_{C}$ thus implies the restriction $v c_{\infty} d / a \phi \ll 1$ on the system for the quasistatic approximation to be valid. Since typically $v c_{\infty} / \phi \sim 10^{-1}$, this criterion is met for a sufficiently small ratio $d / a \leqslant 10^{-1}$.

We also draw attention to the fact that the assumption of local equilibrium at the particle surfaces [Eq. (4)] presumes mass transport across the precipiate-solute interface to be fast on the time scale $\tau_{D}$, thereby restricting the theory to diffusion-controlled (rather than reaction-controlled) growth. ${ }^{3}$

Equations (1)-(5) form the starting point of our analysis of Ostwald ripening in open systems. These equations differ from those given by Voorhees ${ }^{4}$ for the case of a closed system, by the existence of a constant external concentration $c_{0}$ [Eq. (5)]. In a closed system, Eq. (5) must be replaced by a noflux boundary condition on the walls of the vessel containing the solution. In order to avoid complications arising from the finiteness of the system, a boundary condition at infinity,

$$
c \rightarrow c_{0}(t), \quad \text { far from particles }
$$

is adopted instead in the literature-where the time dependent external concentration $c_{0}(t)$ is to be chosen in such a way that conservation of mass in the closed system is ensured. ${ }^{10}$

The source strengths $Q_{t}$ defined in Eq. (2) depend linearly on the values of $c_{\mathrm{eq}}\left(a_{j}\right)-c_{0}(j=1,2, \ldots, N)$ through a relation of the form

$$
Q_{i}=\sum_{j=1}^{N} Z_{i j}\left[c_{\mathrm{eq}}\left(a_{j}\right)-c_{0}\right]
$$

where each element of the transport matrix $Z$ depends on the positions and radii of all the $N$ particles. (For one isolated particle $i, Z_{a}=4 \pi D a_{1}$.) In the next section we shall derive some general properties of the matrix $Z$, essential for our analysis.

\section{GENERAL PROPERTIES OF THE TRANSPORT MATRIX}

From Eqs. (2)-(5) one can show that the transport matrix $Z$ defined in Eq. (7) has the following properties: (i) $Z$ is symmetric, $Z_{t j}=Z_{j l}$; (ii) $Z$ is positive definite, $\Sigma_{l, j} x_{t} Z_{t} x_{j}>0$ for all $x \neq 0$; (iii) in the limit of an infinite system $\Sigma, Z_{t}$ and $\Sigma_{t} Z_{t}$ both vanish.

The symmetry and positive definiteness of $Z$ follow immediately from the identities

$$
\begin{aligned}
\sum_{\iota} \oint_{S_{t}} d S c_{1} \hat{n}_{\imath} \cdot \nabla c_{2} & =-\int_{V_{\mathrm{ex}}} d V\left(\nabla c_{1}\right) \cdot\left(\nabla c_{2}\right) \\
& =\sum_{t} \oint_{S_{t}} d S c_{2} \hat{n}_{t} \cdot \nabla c_{1},
\end{aligned}
$$

valid for any two solutions $c_{1}(\mathbf{r}), c_{2}(\mathbf{r})$ of the Laplace equation (3) which vanish at infinity. [In Eq. (8), $V_{\text {ex }}$ denotes the volume outside the particles.] Indeed, if $c_{1}(\mathbf{r}) \equiv c_{l}^{(1)}, c_{2}(\mathbf{r}) \equiv c_{l}^{(2)}$ on $S_{1}(i=1,2, \ldots, N)$, and $Q_{t}^{(1)}, Q_{i}^{(2)}$ are the corresponding source strengths [defined according to Eq. (2)] we have from Eq. (8),

$$
\sum_{l} c_{l}^{(1)} Q_{l}^{(2)}=\sum_{l} c_{l}^{(2)} Q_{l}^{(1)}
$$

and, taking $c_{1}=c_{2}$,

$$
\sum_{l} c_{l}^{(1)} Q_{1}^{(1)}=D \int_{V_{\mathrm{ex}}} d V\left|\nabla c_{1}\right|^{2}>0 .
$$

Substituting for $Q_{i}=\Sigma_{i} Z_{l j} c_{\text {, }}$ [Eq. (7) with $c_{0}=0$ ] we then obtain the required symmetry and positive definiteness of the transport matrix $Z$.

Before giving a proof of the third property of the transport matrix, it is useful to discuss the relation between this property and the concept of screening of diffusive interactions, developed by Felderhof and Deutch. ${ }^{9}$ Consider to this end the hypothetical situation that $c_{\text {eq }}\left(a_{t}\right)=c_{0}$ for all $i \neq j$, so that $Q_{t}=Z_{i j}\left[c_{\mathrm{eq}}\left(a_{J}\right)-c_{0}\right]$. If $S_{\infty}$ is a large surface which completely encloses the system, then we obtain

$$
\begin{aligned}
\sum_{t} Q_{\imath} & =-D \sum_{t} \oint_{S_{t}} d S \hat{n}_{t} \cdot \nabla c \\
& =-D \oint_{S_{\infty}} d S \hat{n}_{\infty} \cdot \nabla c
\end{aligned}
$$

where $\hat{n}_{\infty}$ is a unit vector perpendicular to $S_{\infty}$ and pointing outward. In a macroscopic system, the perturbation of the concentration caused by particle $j$ is screened by the presence of the other particles, ${ }^{9}$ so that $c(\mathbf{r})-c_{0}$ goes to zero exponentially far from particle $j$. As a consequence, the integral of $\nabla c$ over the large surface $S_{\infty}$ in Eq. (11) gives zero contribution, leading to the vanishing of $\Sigma_{\imath} Z_{\imath \jmath}$ (and also of $\Sigma_{J} Z_{l j}$, by virtue of the symmetry of $Z$ ).

We now proceed to a proof of this third property of the transport matrix. For simplicity, we restrict ourselves here to the so-called monopolar (or point-particle) approximation of diffusive interactions. This restriction can, however, be removed, and the validity of the result is not limited to this approximation. (This is to be expected, since the monopolar approximation describes the long-range form of diffusive interactions, whereas the screening effect results precisely from these long-range contributions.)

In the monopolar approximation the transport matrix can be written in the form (cf. Refs. 6-8)

$$
Z_{\imath \jmath}=4 \pi D a_{j}\left(\delta_{\imath \jmath}-\left\{G(1+G)^{-1}\right\}_{\imath \jmath}\right),
$$

where the matrix $G$ has elements

$$
G_{\imath \jmath}=a_{\imath}\left|\mathbf{R}_{\jmath}-\mathbf{R}_{\imath}\right|^{-1}\left(1-\delta_{\imath \jmath}\right)
$$

It is convenient to write Eq. (12) in an operator representation $^{11}$ 


$$
\begin{aligned}
z\left(\mathbf{r}, \rho \mid \mathbf{r}^{\prime}, \rho^{\prime}\right) & \equiv \sum_{i, j} \delta\left(\mathbf{r}-\mathbf{R}_{i j}\right) \delta\left(\rho-a_{i}\right) Z_{i j} \delta\left(\mathbf{r}^{\prime}-\mathbf{R}_{j}\right) \delta\left(\rho^{\prime}-a_{j}\right) \\
& =4 \pi D \rho^{\prime}\left\{n-n g(1+n g)^{-1} n\right\}\left(\mathbf{r}, \rho \mid \mathbf{r}^{\prime}, \rho^{\prime}\right) .
\end{aligned}
$$

Here $n$ is a diagonal operator,

$$
\begin{aligned}
& n\left(\mathbf{r}, \rho \mid \mathbf{r}^{\prime}, \rho^{\prime}\right)=n(\mathbf{r}, \rho) \delta\left(\mathbf{r}-\mathbf{r}^{\prime}\right) \delta\left(\rho-\rho^{\prime}\right), \\
& n(\mathbf{r}, \rho)=\sum_{i} \delta\left(\mathbf{r}-\mathbf{R}_{i}\right) \delta\left(\rho-a_{i}\right),
\end{aligned}
$$

which gives the microscopic density in position and radius space; the propagator $g$ is a convolution operator in position space,

$$
g\left(\mathbf{r}, \rho \mid \mathbf{r}^{\prime}, \rho^{\prime}\right)=\rho\left|\mathbf{r}^{\prime}-\mathbf{r}\right|^{-1} \theta\left(\left|\mathbf{r}^{\prime}-\mathbf{r}\right|-\rho-\rho^{\prime}\right),
$$

with

$$
\theta(x)=1 \quad \text { if } x>0, \quad \theta(x)=0 \quad \text { if } x<0 .
$$

For later use we record the formula $(k=|\mathbf{k}|)$,

$$
g\left(\mathbf{r}, \rho \mid \mathbf{r}^{\prime}, \rho^{\prime}\right)=(2 \pi)^{-3} \int d \mathbf{k} e^{\lambda \mathbf{k} \cdot\left(\mathbf{r}^{\prime}-\mathbf{r}\right)} 4 \pi \rho \mathbf{k}^{-2} \cos \left[k\left(\rho+\rho^{\prime}\right)\right] .
$$

We proceed by writing the microscopic density field $n(\mathbf{r}, \rho)$ as the sum of its average $n_{0}(\rho)$ and fluctuations $\delta n(\mathbf{r}, \rho)$. (It is assumed here that the system is infinitely large and macroscopically homogeneous in space, so that the average density does not depend on r.) Substituting $n=n_{0}+\delta n$ into Eq. (14), and introducing the renormalized propagator $g^{R}$,

$$
g^{R} \equiv g\left(1+n_{0} g\right)^{-1},
$$

we have the identities

$$
\begin{aligned}
\mathrm{z}\left(\mathbf{r}, \rho \mid \mathbf{r}^{\prime}, \rho^{\prime}\right) & =4 \pi D \rho^{\prime}\left\{n-n g^{R}\left(1-\delta n g^{R}\right)^{-1} n\right\}\left(\mathbf{r}, \rho \mid \mathbf{r}^{\prime}, \rho^{\prime}\right) \\
& =4 \pi D \rho^{\prime}\left\{\left(1-n_{0} g^{R}\right)\left(1+\delta n g^{R}\right)^{-1} n\right\}\left(\mathbf{r}, \rho \mid \mathbf{r}^{\prime}, \rho^{\prime}\right) .
\end{aligned}
$$

The required result now follows immediately from the formula (proven below)

$$
\int d \mathbf{r} \int_{0}^{\infty} d \rho f(\rho) n_{0}(\rho) g^{R}\left(\mathbf{r}, \rho \mid \mathbf{r}^{\prime}, \rho^{\prime}\right)=\overline{\rho f(\rho)} / \bar{\rho}
$$

where $f(\rho)$ is an arbitrary function of $\rho$ and the bar denotes an average with respect to $n_{0}(\rho)$,

$$
\overline{f(\rho)} \equiv \int_{0}^{\infty} d \rho f(\rho) n_{0}(\rho) / \int_{0}^{\infty} d \rho n_{0}(\rho)
$$

Integrating Eq. (20) over $\mathbf{r}$ and $\rho$, and using Eq. (21) with $f \equiv 1$, we find

$$
\int d \mathbf{r} \int_{0}^{\infty} d \rho z\left(\mathbf{r}, \rho \mid \mathbf{r}^{\prime}, \rho^{\prime}\right)=0
$$

which implies the third property, $\Sigma_{i} Z_{i j}=0$, of the transport matrix.

It remains to prove Eq. (21). We do this by showing that $x \equiv \overline{\rho f(\rho)} / \bar{\rho}$ solves the equation

$$
\begin{aligned}
\int d \mathbf{r} & \int_{0}^{\infty} d \rho f(\rho) n_{0}(\rho) g\left(\mathbf{r}, \rho \mid \mathbf{r}^{\prime}, \rho^{\prime}\right) \\
& =x\left(1+\int d \mathbf{r} \int_{0}^{\infty} d \rho n_{0}(\rho) g\left(\mathbf{r}, \rho \mid \mathbf{r}^{\prime}, \rho^{\prime}\right)\right)
\end{aligned}
$$

[from which Eq. (21) then follows in view of definition (19) of the renormalized propagator]. Substitution of expression (18) for $g$ into Eq. (24) gives

$$
\begin{aligned}
\lim _{k \rightarrow 0} & \int_{0}^{\infty} d \rho 4 \pi \rho k^{-2} \cos \left[k\left(\rho+\rho^{\prime}\right)\right] f(\rho) n_{0}(\rho) \\
& =\lim _{k \rightarrow 0} x\left(1+\int_{0}^{\infty} d \rho 4 \pi \rho k^{-2} \cos \left[k\left(\rho+\rho^{\prime}\right)\right] n_{0}(\rho)\right),
\end{aligned}
$$

and hence

$x=\int_{0}^{\infty} d \rho \rho f(\rho) n_{0}(\rho) / \int_{0}^{\infty} d \rho \rho n_{0}(\rho)=\overline{\rho f(\rho)} / \bar{\rho}$,

as we set out to prove.

In the above proof, explicit evaluation of the renormalized propagator $g^{R}$ is avoided. The long-range form of this propagator may be shown [using Eqs. (18) and (19] to be the Deybe-Hückel form discussed in Ref. 9,

$$
g^{R}\left(\mathbf{r}, \rho \mid \mathbf{r}^{\prime}, \rho^{\prime}\right) \approx \rho|\mathbf{r}-\mathbf{r}|^{-1} \exp \left(-\left|\mathbf{r}^{\prime}-\mathbf{r}\right| / \lambda\right),
$$

with the screening length $\lambda$ of diffusive interactions given by

$$
\lambda=(4 \pi \bar{\rho} N / V)^{-1 / 2}[1+\mathscr{O}(N / V)]
$$

( $N$ is the total number of particles, $V$ the volume of the system). In terms of the volume fraction $\phi \equiv \frac{4}{3} \tau \overline{\rho^{3}} N / V$ of the precipitate we have the useful approximation for dilute systems

$$
\lambda \approx \bar{\rho}\left(3 \phi(\bar{\rho})^{3} / \bar{\rho}^{3}\right)^{-1 / 2} \approx \bar{\rho} / \sqrt{\phi} .
$$

We conclude this section by recording a general formula following from Eqs. (20) and (21) [using also the symmetry $\left.z\left(\mathbf{r}, \rho \mid \mathbf{r}^{\prime}, \rho^{\prime}\right)=z\left(\mathbf{r}^{\prime}, \rho^{\prime} \mid \mathbf{r}, \rho\right)\right]$

$$
\begin{aligned}
\int d \mathbf{r}^{\prime} & \int_{0}^{\infty} d \rho^{\prime} z\left(\mathbf{r}, \rho \mid \mathbf{r}^{\prime}, \rho^{\prime}\right) f\left(\rho^{\prime}\right) \\
= & 4 \pi D \rho \int d \mathbf{r}^{\prime} \int_{0}^{\infty} d \rho^{\prime}\left[f\left(\rho^{\prime}\right)-\overline{\rho f(\rho)} / \bar{\rho}\right] \\
& \times\left\{\left(1+\delta^{n} g^{R}\right)^{-1} n\right\}\left(\mathbf{r}^{\prime}, \rho^{\prime} \mid \mathbf{r} \rho\right)
\end{aligned}
$$

which we shall need later on.

\section{EQUIVALENCE OF OPEN AND CLOSED SYSTEMS}

As discussed in Sec. II [see Eqs. (1), (4), and (7)] the diffusion-controlled coarsening (Ostwald ripening) of a precipitate is described by the following evolution equation for the radii $a_{t}$ of the precipitated particles:

$$
\frac{d a_{i}}{d t}=-\frac{1}{4 \pi} v c_{\infty} a_{i}^{-2} \sum_{j} Z_{i j}\left(\Delta+d / a_{j}\right),
$$

where $v$ is the molar volume of the precipitate, $c_{\infty}$ the molar solute concentration in equilibrium with a plane surface, and $d$ the capillary length of this problem ${ }^{3} ; \Delta \equiv\left(c_{\infty}-c_{0}\right) / c_{\infty}$ equals the difference between $c_{\infty}$ and the external concentration $c_{0}$ (see below), relative to $c_{\infty}$.

In an open system immersed in a solute reservoir, $\Delta$ is a constant determined by the concentration $c_{0}$ of the solute in the reservoir [Eq. (5)]. In a closed system, on the other hand, $\Delta$ is taken to be time dependent so as to ensure conservation of mass in the system [Eq. (6)]. ${ }^{10}$ The equivalence of these two situations in the limit of infinite systems results from the 
third property of the transport matrix $Z$ derived in Sec. III, which states that $\Sigma_{j} Z_{i j}=\Sigma_{i} Z_{i j}=0$ in this limit. Therefore, the evolution of the system, as determined by Eq. (31), is independent of $\Delta$ and thus identical for open and closed infinite systems.

This remarkable irrelevance of the external concentration has its physical origin in the phenomenon of screening ${ }^{9}$ (see Sec. III); the bulk of the system is, in effect, screened from the solute concentration field in the reservoir by the diffusive interactions between the particles. The influence of the reservoir is only felt in a penetration layer at the boundaries of the system of depth $\lambda \approx a / \sqrt{\phi}$, which is the screening length of the diffusive interactions [with $\phi$ the volume fraction of the precipitate and $a$ the average particle radius, cf. Eq. (29].

The above result of equivalence of open and closed systems holds strictly in the limit of infinite system size. We expect, however, the influence of the finiteness of the system to be negligible as long as the system size $L$ is much larger than the screening length $\lambda$. We shall deal with this issue in the remainder of this section.

Two effects should be distinguished which cause an increase of the ratio $\lambda / L$ from its initial value at time $t=0$. First, coarsening of the precipitate tends to increase the average radius at constant volume fraction, leading to an increase of $\lambda \approx \mathrm{a} / \sqrt{\phi}$. From the Lifshitz-Slyosov-Wagner growth law $^{2,3}$ we obtain the estimate

$$
\lambda(t) \approx \lambda(0)\left(1+t / \tau_{C}\right)^{1 / 3},
$$

with the coarsening time scale $\tau_{C}=a(0)^{3} / D v c_{\infty} d(D$ is the diffusion coefficient of the solute). Here, and in the following estimate, numerical coefficients of order unity are disregarded.

Second, in an undersaturated reservoir $\left(c_{0}<c_{\infty}, \Delta>0\right)$, the precipitate at the boundaries of the system dissolves, causing the effective size of the system to decrease. Let $V_{p}$ be the volume of the precipitate and $S_{p}$ the surface area exposed to the solute concentration field in the reservoir. Assuming a system of approximately spherical shape (with radius $L$ ), we have $V_{p}=\frac{4}{3} \pi L^{3} \phi, S_{p}=4 \pi L^{2} \lambda 3 \phi / a(=$ total surface area of particles in penetration layer of depth $\lambda$ ). We furthermore determine the average molar flux $\Psi$ of solute from the system to the reservoir by $\Psi=D\left(c_{\infty}-c_{0}\right) \times$ average curvature $=D\left(c_{\infty}-c_{0}\right) \frac{1}{2} \partial S_{p} / \partial V_{p}=D c_{\infty} \Delta 3 \lambda / a L$ (the factor $\partial S_{p} / \partial V_{p}$ is the quotient of surface and volume change with an increase of length scale). Inserting the above expressions into the balance equation $d V_{p} / d t=-v S_{p} \Psi$, we find

$$
\frac{d}{d t} L(t)=-9 D v c_{\infty} \Delta(\lambda / a)^{2} / L(t),
$$

which gives upon integration the estimate (using $\lambda / a \approx 1 / \sqrt{\phi}=$ constant)

$$
L(t) \approx L(0)\left(1-t / \tau_{R}\right)^{1 / 2},
$$

with

$$
\tau_{R}=\phi L(0)^{2} / D v c_{\infty} \Delta .
$$

We are now in the position to determine the time scale on which the effect of a finite system size may be neglected.
From Eqs. (32) and (34) we obtain the requirement $\lambda(t)$ $L(t) \ll 1$ in the form

$$
\frac{\lambda(t)}{L(t)} \approx \frac{\lambda(0)}{L(0)} \frac{\left(1+t / \tau_{C}\right)^{1 / 3}}{\left(1-\kappa[\lambda(0) / L(0)]^{2} t / \tau_{C}\right)^{1 / 2}} \ll 1,
$$

with $\kappa \equiv \Delta a(0) / d$. If the initial ratio $\lambda(0) / L(0)$ of screening length to system size is sufficiently small, then this ratio increases in times as $\left(t / \tau_{C}\right)^{1 / 3}$; a tenfold increase takes a time $10^{3} \tau_{C}$, which is already much longer than the characteristic time scale $\tau_{C}$ of the coarsening process.

We conclude with a numerical example. Consider an undersaturated reservoir with $c_{0} \ll c_{\infty}(\Delta \sim 1)$ and a precipitate with $a(0) \sim 10^{-6} \mathrm{~cm}, d \sim 10^{-7} \mathrm{~cm}, L(0) \sim 1 \mathrm{~cm}$, and $\phi \sim 10^{-2}$. Then we have $\lambda(0) \approx a(0) / \sqrt{\phi} \sim 10^{-5} \mathrm{~cm}$ and $\kappa \sim 10$. If we regard a ratio $\lambda(t) / L(t) \sim 10^{-3}$ as sufficiently small for finite system size effects to be negligible, then we find from Eq. (35) that these effects may indeed be neglected for times $t \lesssim 10^{6} \tau_{C}$.

\section{CONCLUDING REMARKS}

In this last section we present some additional consequences of the general properties of the coarsening equations derived in Sec. III.

First of all, we wish to show how these properties afford a direct microscopic derivation of the evolution equation for the particle-size distribution function in the case of a dilute system. Consider to this end the equation for the time derivative of the microscopic density field of particles $n(\mathbf{r}, \rho, t)=\Sigma_{i} \delta\left(\mathbf{r}-\mathbf{R}_{i}\right) \delta\left[\rho-a_{i}(t)\right]$, as it follows from Eq. (31),

$$
\begin{aligned}
& \frac{\partial}{\partial t} n(\mathbf{r}, \rho, t)=\frac{1}{4 \pi} v c_{\infty} \frac{\partial}{\partial \rho} \rho^{-2} \int d \mathbf{r}^{\prime} \\
& \quad \times \int_{0}^{\infty} d \rho^{\prime} z\left(\mathbf{r}, \rho \mid \mathbf{r}^{\prime}, \rho^{\prime}\right)\left(\Delta+d / \rho^{\prime}\right), \quad \rho>0 .
\end{aligned}
$$

The kernel $z\left(\mathbf{r}, \rho \mid \mathbf{r}^{\prime}, \rho^{\prime}\right)$ has been defined in Eq. (14). Using formula (30) of Sec. III we obtain from Eq. (36), in the limit of zero volume fraction of the precipitate, an equation for the time evolution of the particle-radius distribution function $n_{0}(\rho, t)$ [which is the average of $n(\mathbf{r}, \rho, t)$ in a spatially homogeneous system]. We find ${ }^{12}$

$$
\frac{\partial}{\partial t} n_{0}(\rho, t)=-D v c_{\infty} d \frac{\partial}{\partial \rho} \rho^{-2}(\rho / \bar{\rho}(t)-1) n_{0}(\rho, t),(37)
$$

with $\bar{\rho}(t)$ the average radius. In agreement with the general argument presented in the previous section, $\Delta$ no longer appears in this equation, which holds therefore for open and closed infinite systems alike.

In the case of a closed system, Eq. (37) has been obtained by Wagner ${ }^{3}$ (on the basis of a mean-field theory) and by Tokuyama and Kawasaki ${ }^{7}$ (from a microscopic starting point). For corrections resulting from the finite volume fraction of the particles, we refer to the latter paper ${ }^{7}$ (cf. also Ref. 13) and to the work of Marqusee and Ross ${ }^{14}$; for a solution of Eq. (37), see Ref. 3.

Equation (37) differs from the evolution equation for a closed system given by Lifshitz and Slyosov, ${ }^{2}$ in that their equation allows for a variation in time of the volume fraction 
$\phi$ of the precipitate, whereas Eq. (37) implies that $\phi \equiv \frac{4}{3} \pi \int_{0}^{\infty} d \rho \rho^{3} n_{0}(\rho, t)$ is constant in time. The solutions of both equations coincide, however, in the regime of validity of the quasistatic diffusion equation [Eq. (3)]. To see this, note that the Lifshitz-Slyosov equation ${ }^{2}$ predicts a total increase $\Delta \phi$ in the volume fraction of order $\Delta \phi \approx v\left[c_{\mathrm{eq}}(a)\right.$ $\left.-c_{\infty}\right]=v c_{\infty} d / a(a$ is the initial average radius of the particles). The criterion $\Delta \phi / \phi \ll 1$, therefore, coincides with the requirement $v c_{\infty} / a \phi \ll 1$ necessary for the quasistatic approximation to be valid, cf. Sec. II. (For a related discussion of this point, see Ref. 15.)

It follows immediately from Eq. (37) that the surface to volume ratio of the precipitate is a decreasing function of time. This can be shown to be a general result, not restricted to the dilute regime. First, for the time derivative of the total volume we have the expression [cf. Eq. (31)]

$$
\frac{d}{d t} \sum_{i} \frac{4}{3} \pi a_{i}^{3}=-v c_{\infty} \sum_{i, j} Z_{i j}\left(\Delta+d / a_{j}\right)=0,
$$

which vanishes for a macroscopic system by virtue of the third property of the transport matrix (see Sec. III). Second, for the time derivative of the total surface area we have

$\frac{d}{d t} \sum_{i} 4 \pi a_{i}^{2}=-2 v c_{\infty}\left(\Delta \sum_{i, j} \frac{1}{a_{i}} Z_{\imath j}+d \sum_{i, j} \frac{1}{a_{i}} Z_{i j} \frac{1}{a_{j}}\right)<0$.

The first term between brackets on the right-hand side of $E q$. (39) vanishes (see above), whereas the second term is positive because of the positive definiteness of $Z$ (Sec. III). That the surface to volume ratio of the precipitate decreases in time, then follows from Eqs. (38) and (39)-irrespective of the volume fraction.

These observations further illustrate the importance of the general relations of Sec. III, which place the theory of
Ostwald ripening for open systems on as firm a footing as the well-established theory for closed systems.

\section{ACKNOWLEDGMENTS}

We thank M. LeVan, J. A. Marqusee, and J. Reiter for valuable discussions.

${ }^{1}$ W. Ostwald, Analytische Chemie, 3rd ed. [Engelmann, Leipzig, 1901), p. 23.

${ }^{2}$ I. M. Lifshitz and V. V. Slyosov, J. Phys. Chem. Solids 19, 35 (1961).

${ }^{3} \mathrm{C}$. Wagner, Z. Elektrochem. 65, 581 (1961).

${ }^{4}$ P. W. Voorhees, J. Stat. Phys. 38, 231 (1985).

${ }^{5} \mathrm{G}$. Venzl, Phys. Rev. A 31, 3431 (1985). The model of solute transport proposed by Venzl is, however, unsatisfactory, due to neglect of screening of diffusive interactions (which plays an essential role, as shown in the present paper).

${ }^{6} \mathrm{~J} . \mathrm{J}$. Weins and J. W. Cahn, in Sintering and Related Phenomena, edited by G. C. Kuczynski (Plenum, New York, 1973).

${ }^{7} \mathrm{M}$. Tokuyama and K. Kawasaki, Physica A 123, 386 (1984).

${ }^{8}$ P. W. Voorhees and M. E. Glicksman, Acta Metall. 32, 2001, 2013 (1984).

${ }^{9}$ B. U. Felderhof and J. M. Deutch, J. Chem. Phys. 64, 4551 (1976).

${ }^{10}$ Two different time dependencies of the external concentration $c_{0}(t)$ have been used in the literature, following Refs. 2 and 3, respectively: (1) $c_{0}(t)$ such that $v c_{0}+\phi=$ constant; (2) $c_{0}(t)$ such that $\phi=$ constant. In the regime of validity of the quasistatic diffusion equation these two prescriptions coincide, cf. the discussion following Eq. (37) in Sec. III.

${ }^{11}$ We adapt here a formalism used previously in the context of a hydrodynamic many-body problem, see C. W. J. Beenakker and P. Mazur, Physica A 120, 388 (1983).

${ }^{12}$ Equation (37) results from the average of Eq. (36) by virtue of Eq. (30) [with $\left.f\left(\rho^{\prime}\right)=\Delta+d / \rho^{\prime}\right]$; the inverse operator appearing between braces on the right-hand side of Eq. (30) may be approximated by unity in the dilute regime.

${ }^{13}$ M. Tokuyama, Y. Enomoto, and K. Kawasaki (preprint).

${ }^{14}$ J. A. Marqusee and J. Ross, J. Chem. Phys. 80, 536 (1984).

${ }^{15}$ J. A. Marqusee and J. Ross, J. Chem. Phys. 79, 373 (1983). 